\title{
Map Projection Article on Wikipedia
}

\author{
Miljenko Lapaine ${ }^{\mathrm{a}, *}$ \\ ${ }^{a}$ University of Zagreb, Faculty of Geodesy, mlapaine@geof.hr \\ * Corresponding author
}

\begin{abstract}
People often look up information on Wikipedia and generally consider that information credible. The present paper investigates the article Map projection in the English Wikipedia. In essence, map projections are based on mathematical formulas, which is why the author proposes a mathematical approach to them. Weaknesses in the Wikipedia article Map projection are indicated, hoping it is going to be improved in the near future.
\end{abstract}

Keywords: map projection, Wikipedia

\section{Introduction}

Not so long ago, if we were to find a definition of a term, we would have left the table with a matching dictionary, a lexicon or an encyclopaedia, and in that book asked for the term. However, things have changed. There is no need to get up, it is enough with some search engine to search that term on the internet. On the monitor screen, the search term will appear with the indication that there are thousands or even more of them. One of the most famous encyclopaedias on the internet is certainly Wikipedia. Wikipedia is a free online encyclopaedia, created and edited by volunteers around the world.

According to Wikipedia (2018a) "The reliability of Wikipedia (predominantly of the English-language edition) has been frequently questioned and often assessed. The reliability has been tested statistically, through comparative review, analysis of the historical patterns, and strengths and weaknesses inherent in the editing process unique to Wikipedia. Incidents of conflicted editing, and the use of Wikipedia for 'revenge editing' (inserting false, defamatory or biased statements into biographies) have attracted publicity." Nevertheless, many people usually look and more or less trust to Wikipedia. That is why this paper will investigate the article Map projection at the English-language edition of Wikipeadia (2018b).

In some parts of the world cartography belongs to geography, in some other parts to geodesy. According to my experience as a longtime university professor, most geographers and geodesists do not like mathematics. If you look at any of the textbooks on map projections, you will find plenty of mathematical formulas. For this reason, I advocate access to map projections from a point of view close to math. We should remember that in the not-toodistant past, and in some countries even today, the synonym of studying map projections was known as mathematical cartography.

\section{Map Projection Article on Wikipedia}

The contents of the article Map projection at the Englishlanguage edition of Wikipedia reads:

1 Background

2 Metric properties of maps

2.1 Distortion

3 Construction of a map projection

3.1 Choosing a projection surface

3.2 Aspect of the projection

3.3 Notable lines

3.4 Scale

3.5 Choosing a model for the shape of the body

4 Classification

5 Projections by surface

5.1 Cylindrical

5.2 Pseudocylindrical

5.3 Hybrid

5.4 Conic

5.5 Pseudoconic

5.6 Azimuthal (projections onto a plane)

6 Projections by preservation of a metric property

6.1 Conformal

6.2 Equal-area

6.3 Equidistant

6.4 Gnomonic

6.5 Retroazimuthal

6.6 Compromise projections

7 Which projection is best?

8 See also

9 References

10 External links

The following text warns of weaknesses in that article with the desire to improve it in the future. 


\subsection{Definition of Map Projection}

After a medieval illustration of the Ecumene from 15th century at the article Map projection on Wikipedia the definition of map projection follows: „A map projection is a systematic transformation of the latitudes and longitudes of locations from the surface of a sphere or an ellipsoid into locations on a plane." This definition is taken from a book by Snyder and Voxland (1989).

Snyder (1993) gives this definition: "The systematic representation of all or part of the surface of a round body, especially the earth, onto a flat or plane surface is called a map projection."

Map Projections (USGS 2000) starts with the definition: "A map projection is used to portray all or part of the round Earth on a flat surface". There is another definition in a small glossary located at the end of this publication " A map projection is a systematic representation of a round body such as the Earth on a flat (plane) surface".

In the National Atlas of the United States, which since 2014 has no longer been directly available on the Internet but can be found at another address (Wayback Machine 2014), map projections are defined in the following way: "A map projection is a way to represent the curved surface of the Earth on the flat surface of a map."

These three definitions of map projections (Snyder 1993, USGS 2000, Wayback Machine 2014) are very similar. What is common to them all is that they are about mapping (systematic representation or a way to represent).

Mapping is one of the basic terms in mathematics. To recall, two non-empty sets, $X$ and $Y$, are given. By mapping we understand the rule that each element $x$ from $X$ joins element $y$ from $Y$. So, we write $f: X \rightarrow Y$. Element $y$ is the image of $x$, so we write $y=f(x)$. Set $X$ is the domain of mapping $f$, while set $Y$ is codomain.

The difference between the three definitions of map projection is in the domain (a round body or curved surface or surface of a round body) and in the codomain (a flat (plane) surface or flat surface or a flat or plane surface).

Mathematicians differentiate between a body and a surface. A boundary of a disk is a circle. Similarly, a boundary of a ball is a sphere. Since map projections are mappings from a surface (usually a sphere or an ellipsoid) into a plane, a body should not be taken as the domain in the definition of map projection.

Furthermore, mathematicians differentiate between a plane and a flat surface. The plane is a fundamental concept in geometry; a flat surface in three-dimensional space, which expands to infinity in every direction. If it is flat, it means that an infinite number of straight-lines pass through each of its points, which it completely contains. Or "A flat surface on which a straight line joining any two points on it would wholly lie." From another point of view, "A regular surface and special class of minimal surface are flat surfaces if the Gaussian curvature vanishes everywhere. A tangent developable, generalized cone, and generalized cylinder are all flat surfaces". (Weisstein 2017). From this, we can conclude that a plane and a flat surface are not synonyms. Moreover, since conical and cylindrical surfaces are flat surfaces, it would make sense in the definition of map projections for them to be taken as codomains too, if indeed the mapping was either on the cone or cylinder mantle, which is generally not the case. It would also be contrary to the explanation regularly encountered in the introduction of map projections, that the globe occupies a lot of space, is difficult to translate, is financially demanding to produce and update, and even the largest globe made to a very small scale shows relatively few details. Of course, everything said about globes in the form of spheres, such as those we are accustomed to, also applies to globes in the form of cones or cylinders. Consequently, a map is not displayed on a flat surface (a conical or cylindrical surface) but in a plane.

Let us look at the adjective systematic in some definitions of map projections. If a systematic approach is understood to mean "an approach that is methodical, repeatable and able to be learned by a step-by-step procedure", that is acceptable. However, the adjective can be used in a variety of ways, so it might be better to replace it with planned or organized. If we keep the definition of map projection as a representation, then we need to give it an adjective to distinguish it from a photograph or another image. If we use mapping in the meaning of the mathematical term, then there is no need for another word.

To conclude, the domain of map projection should be a surface rather than a body. For the codomain of map projection, the concept of a plane rather than a flat surface should be used. Instead of representation it is better to say mapping, assuming that the concept of mapping is known from mathematics. It should be, because it has always been around in education. So why not use it, when it is so neatly linked to the word map?

If we look at the Russian literature we can find the following definitions of map projections. The Russian term otobrazhenie was translated as mapping.

"Any mathematically defined way of mapping all or part of the surface of an earth's ellipsoid or earth's sphere on a plane is called a map projection" (Kavrayskiy, 1958).

"... when creating maps, they resort to map projections, in which the surface is mapped on a plane according to certain mathematical laws" (Vakhrameeva et al., 1986).

"1. Map projection is called a mathematically expressed way of mapping the Earth's surface or other celestial bodies taken as an ellipsoid, sphere, or some other regular surface in a plane; 2. Map projection is called a way of determining the one-to-one relationship between the points of a surface being mapped and the plane" (Bugayevskiy, 1998).

"A map projection is a mathematically defined mapping of the surface of an ellipsoid or sphere on a map plane" (Serapinas, 2005).

The proposed definition of map projection in this paper would then be as follows:

A map projection is the mapping of a curved surface, especially a sphere or ellipsoid, into a plane. 
“... most of the Map projection article in Wikipedia assumes that the surface to be mapped is a sphere. In reality, the Earth and other large celestial bodies are generally better modeled as oblate spheroids, whereas small objects such as asteroids often have irregular shapes. These other surfaces can be mapped as well. Therefore, more generally, a map projection is any method of "flattening" a continuous curved surface onto a plane." Everything is fine in this paragraph at the end of the chapter Background of the article Map projection on Wikipedia, only that word flattening strongly resembles ironing, so term mapping would be a much happier choice.

\subsection{Leonhard Euler}

The chapter Background in the article Map projection on Wikipedia starts with these words: „Maps can be more useful than globes in many situations: they are more compact and easier to store; they readily accommodate an enormous range of scales; they are viewed easily on computer displays; they can facilitate measuring properties of the region being mapped; they can show larger portions of the Earth's surface at once; and they are cheaper to produce and transport. These useful traits of maps motivate the development of map projections.

However, Carl Friedrich Gauss's Theorema Egregium proved that a sphere's surface cannot be represented on a plane without distortion." The last sentence is correct, but injustice has been done to a prominent mathematician, physicist and cartographer Leonhard Euler (1707-1783). He has authored more than 700 works (books and articles) from mathematics, celestial mechanics, hydraulics and cartography. In 1777 he published three papers on map projections:

De repraesentatione superficiei sphaericae super plano

De projectione geographica superficiei sphaericae

De projectione geographica De Lisliana in mappa generali imperii russici usita

In the first of them, "On mapping the sphere in the plane" he gave the first formal proof of the impossibility of mapping the sphere into the plane without any distortion (Euler, 1777). In his original form, his papers were written in Latin, re-released in 1955 in Switzerland under Euler's Opera Omnia, translated and published in 1898 in Germany, in Poland in 1949, in 1959 in Russia, in England in 1965 and in Croatia in 2014. C. F. Gauss was born in 1777, and that same year, L. Euler published proof of the impossibility of mapping the sphere in the plane without any distortion. Therefore, the championship belongs to Euler and should not be overwhelmed.

\subsection{Coordinates}

The third paragraph of chapter Background of the article Map projection on Wikipedia reads "Projection is not limited to perspective projections, such as those resulting from casting a shadow on a screen, or the rectilinear image produced by a pinhole camera on a flat film plate. Rather, any mathematical function transforming coordinates from the curved surface to the plane is a projection. Few projections in actual use are perspective." The second of cited sentences is about coordinate transformation. In fact, the coordinates themselves are not the beginning or the end of something, except in mathematical exercises. These are numbers that define the position of a point on a curve, in a plane, or in space. Coordinates allow or facilitate an analytical record of the relationship between points in space. So, if we want to define a map projection as a transformation, and this is just another word for mapping in this context, then its domain of the definition and the area of the mapping value (codomain) should be carefully defined.

\subsection{Distortions}

The most important subject in the theory of map projections are distortions. Unfortunately, this topic is elaborated in the article Map projection of Wikipedia as the shortest one. Is consist of three sentences altogether speaking of the Tissot's indicatrix or ellipse of distortion. These three sentences are understandable, although the illustration was taken from Mercator projection showing circles of distortion, because this projection is conformal. It would be better to illustrate a general case with ellipses of distortion.

\subsection{Construction of a map projection}

Wikipedia (2018b) says: "Some of the simplest map projections are literal projections, as obtained by placing a light source at some definite point relative to the globe and projecting its features onto a specified surface. This is not the case for most projections, which are defined only in terms of mathematical formulae that have no direct geometric interpretation. However, picturing the light source-globe model can be helpful in understanding the basic concept of a map projection."

First, projections obtained by placing a light source at some definite point relative to the globe and projecting its features onto a specified surface are not the simplest projections. For example, it is not possible to obtain equidistant cylindrical nor equidistant azimuthal projection by using a light source as explained in the last sentence. Second, if Wikipedia says that projections defined only in terms of mathematical formulae have no direct geometric interpretation, then Wikipedia (2018c) did not aware of its own web-site on geometry, where one can read: "Geometry is a branch of mathematics concerned with questions of shape, size, relative position of figures, and the properties of space." In other words, all map projections belong to geometry, regardless of the way of its definition. Third, there is no proof that "picturing the light source-globe model can be helpful in understanding the basic concept of a map projection". On contrary, the research reported by Downs and Liben (1991), however, suggested that using the light-andshadows in concert with developable surfaces is not necessarily well-matched to the cognitive abilities of all students. 


\subsection{Choosing a projection surface}

Wikipedia (2018b) says: „A surface that can be unfolded or unrolled into a plane or sheet without stretching, tearing or shrinking is called a developable surface. The cylinder, cone and the plane are all developable surfaces. The sphere and ellipsoid do not have developable surfaces, so any projection of them onto a plane will have to distort the image."

First, how one can unfold or unroll a plane into a plane? What does developing a plane in the plane mean? Development is isometry, so from the cartographic point of view, nothing would change, and we do not need a plane as a developable surface. Second, if Wikipedia says: "The sphere and ellipsoid do not have developable surfaces" then something is strange, because a sphere and an ellipsoid are surfaces itself. In fact "A sphere is a perfectly round geometrical object in three-dimensional space that is the surface of a completely round ball." (Wikipedia 2018d); "An ellipsoid is a surface that may be obtained from a sphere by deforming it by means of directional scalings, or more generally, of an affine transformation." (Wikipedia 2018e). To conclude, the reason why any map projection will have to distort the image was explained by Euler (1777) and others after him, and it has nothing to do with developable surfaces.

Wikipedia (2018b) says: "One way of describing a projection is first to project from the Earth's surface to a developable surface such as a cylinder or cone, and then to unroll the surface into a plane. While the first step inevitably distorts some properties of the globe, the developable surface can then be unfolded without further distortion." Yes, this is true, but completely unimportant in general, because most projections are defined only in terms of mathematical formulae. Moreover, there is an illustration showing the Miller cylindrical projection with the made-up caption saying it maps the globe onto a cylinder (Fig. 1). But there is no cylinder at all in the definition of Miller cylindrical projection. It is enough to look at Miller's original paper (Miller 1942).

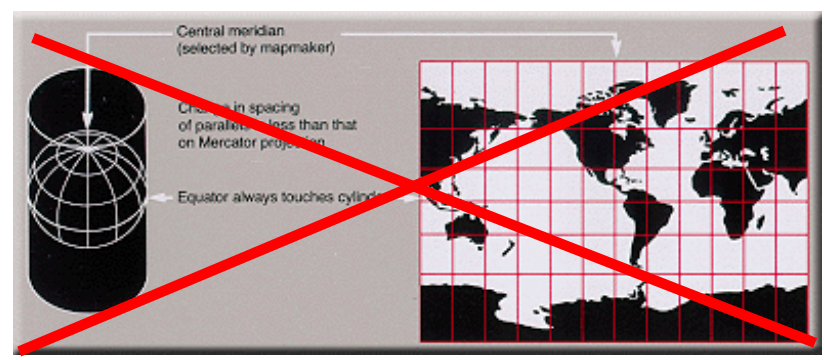

Figure 1. Illustration of the Miller projection with fictitious and erroneous caption: „A Miller cylindrical projection maps the globe onto a cylinder." Public Domain, https://commons. wikimedia.org/w/index.php?curid $=61903$

\subsection{Aspect of the projection}

Wikipedia (2018b) defines aspect by using the placement of a developable surface relative to the globe. That means that aspect exists only for map projections defined by developable surfaces. But this is not true, because there exist many projections, e.g. pseudocylindricals, which are not defined by developable surfaces but can be represented in transverse and oblique aspects.

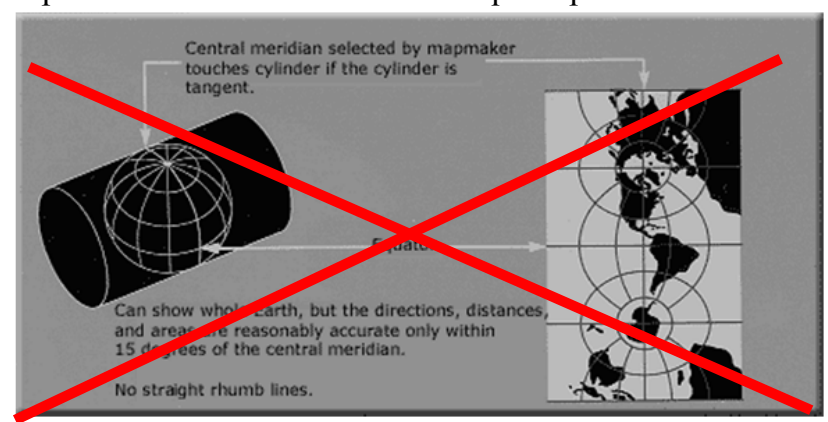

Figure 2. Illustration of transverse Mercator projection with two mistakes. First, there is no cylinder in the definition of Mercator projection. Second, Mercator projection cannot show whole Earth, because it maps a globe onto an infinite long strip.

It would be better to define aspect as proposed by Lapaine and Frančula (2016). They showed that the aspect can be defined without using developable surface story and can be applied to any map projection.

Moreover, there is an illustration in Wikipeadia (2018b) showing transverse Mercator projection, and saying "Can show whole Earth" (Fig. 2). But everybody should know that this is impossible for any aspect of the Mercator projection, because it maps a globe onto an infinite long strip.

\subsection{Notable lines and zero distortion}

Developable surfaces are the most important objects of interest of Wikipedia (2018b)! The chapter Notable lines starts with explanation that developable surfaces may be either tangent or secant to the sphere or ellipsoid. It follows: "Tangent and secant lines (standard lines) are represented undistorted." The last statement is not correct. First, there are distinct kinds of distortion in map projections. If standard lines are undistorted, then there is zero distortion of length, area and angle at any point of such a line. But, this is not the case as shown by Lapaine (2017a, b).

\subsection{Classification}

The chapter Classification in Map projection site on Wikipedia (2018b) starts also with developable surfaces: "A fundamental projection classification is based on the type of projection surface onto which the globe is conceptually projected." The statement is not correct, because map projections are not mappings onto developable surfaces, in general. Let us remind, a map projection is the mapping of a curved surface, especially a sphere or ellipsoid, into a plane. Naturally the question arises: Why cylindrical projections has a name cylindrical? Cylindrical projection is not a mapping onto a cylinder, but the map produced in a cylindrical projection can be bend into a cylindrical surface. Similarly, conic projection is not a mapping onto a cone, but the map produced in a conic projection can be bend into a conic surface. 


\subsection{Projection by surface}

The chapter Projection by surface in Map projection article on Wikipedia (2018b) starts again with developable surfaces: "The three developable surfaces (plane, cylinder, cone) provide useful models for understanding, describing, and developing map projections. However, ..." And then, finally, there are citations by Lee (1944) who explained why cylinders and cones have given rise to much misunderstanding. Despite that clear explanation, Wikipedia continues "If maps were projected as in light shining through a globe onto a developable surface, then ...". Almost unbelievable developable surfaces survive!

A few sentences further, we can read: "If you rotate the globe before projecting then the parallels and meridians will not necessarily still be straight lines. Rotations are normally ignored for the purpose of classification." The last statement is obviously wrong, because there is a classification of map projections according to aspect into normal, transversal and oblique, regardless of the definition of aspect.

\subsection{Cylindrical}

The chapter on cylindrical projections in Map projection article of Wikipedia (2018b) starts with the illustration of the Mercator projection (Fig. 3). Unfortunately, the illustration shows a cylinder, although Mecator did not use any developable surface.

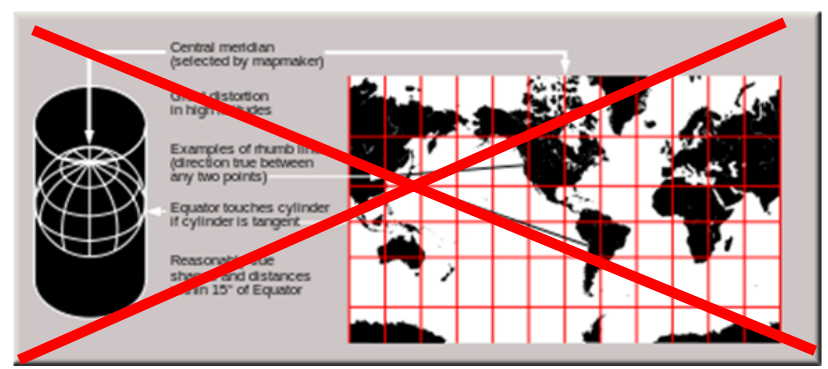

Figure 3. Illustration of normal aspect Mercator projection, Wikipedia (2018b). Wikipedia do not know that Mercator did not use any cylinder for his projection.

The definition of cylindrical projection follows: "A "normal cylindrical projection" is any projection in which meridians are mapped to equally spaced vertical lines and circles of latitude (parallels) are mapped to horizontal lines." The definition is as usual, but it does not include the general case, when mapped meridians are not equally spaced. Due to obvious obsession with developable surfaces the text continues: "The mapping of meridians to vertical lines can be visualized by imagining a cylinder whose axis coincides with the Earth's axis of rotation. This cylinder is wrapped around the Earth, projected onto, and then unrolled." In general, cylindrical projections are not projections onto a cylinder surface. Furthermore, such visualization certainly does not help understanding the cylindrical projections in which the meridian images are not spaced equally.

At the end of the chapter on cylindrical projection, one can read: "In the first case (Mercator), the east-west scale always equals the north-south scale. In the second case (central cylindrical), the north-south scale exceeds the east-west scale everywhere away from the equator. Each remaining case has a pair of secant lines - a pair of identical latitudes of opposite sign (or else the equator) at which the east-west scale matches the north-south-scale." The last statement shows that Wikipedia do not know that secant lines and zero distortion lines are two different concepts.

\subsection{Pseudocylindrical}

Nobody knows why the chapter on pseudocylindrical projections appears in Projection by surface chapter of the Map projection article on Wikipedia (2018b). Which surface is wrapped around the sphere, projected onto, and then unrolled? In other words, what is a pseudocylinder surface and is it a developable surface?

The chapter starts with an illustration of sinusoidal projection and its relation to the interrupted version. It would be better to start with some usual pseudocylindrical projections, like Mollweide, Eckert IV or Robinson, and then mention the possibility of interrupted projections.

In the definition of pseudocylindrical projections Wikipedia says: "Along parallels, each point from the surface is mapped at a distance from the central meridian that is proportional to its difference in longitude from the central meridian". This statement is not correct, because there are pseudocylindrical projections where meridians are not equally spaced along a given parallel (see e.g. Snyder 1977).

\subsection{Hybrid}

In my opinion, in the chapter Hybrid of Map projection article of Wikipedia (2018b) should be mentioned other possibilities like combined projections, blended projections etc. HEALPix can be just one example.

\subsection{Conic}

The chapter on conic projections in Map projection article of Wikipedia (2018b) starts with the illustration of the Albers projection (Fig. 4). The illustration suggests that images of two secant parallels are standard parallels, i.e. parallels with zero distortion. This is not true in conic projecions in general, and especially in Albers projection. The proof is simple. Let us notice two parallels on the sphere with radius $R$, and let $\varphi_{1}$ and $\varphi_{2}$ are corresponding latitudes $0<\varphi_{1}<\varphi_{2}<90^{\circ}$. The shortest distance between these two parallels equals $2 R \sin \frac{\varphi_{2}-\varphi_{1}}{2}$. If these two parallels are standard paralles in Albers projection, then the shortest distance between the images of these to parallels in the plane of projection equals $2 R \tan \frac{\varphi_{2}-\varphi_{1}}{2}$. Everybody see that the two distances are different. So, we can conclude that a map produced in Albers projection and bent into conical surface cannot be placed so that cut the sphere along two chosen parallels. 


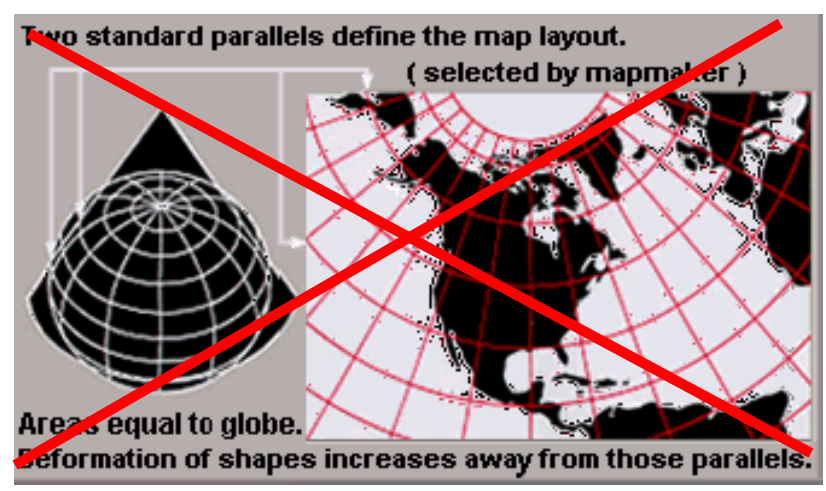

Figure 4. Illustration of Albers projection, Wikipedia (2018b). Wikipedia do not know that secant lines and zero distortion lines are two different lines, in general.

The text that follows in the chapter Conic of the Map projection article in Wikipedia (2018b) describes standard parallels as secant lines, which is generally wrong, and we have shown it in the example of Albers conic projection.

\subsection{Pseudoconic}

There is no definition on pseudoconic projections in the chapter Pseudoconic of the Map projection article in Wikipedia (2018b). Moreover, nobody knows why the chapter on pseudoconic projections appears in Projection by surface chapter. Which surface is wrapped around the sphere, projected onto, and then unrolled? In other words, what is a pseudoconic surface and is it a developable surface?

When speaking about Bonne projection, it should be added that distances along each parallel and along the central meridian have zero distortion. Furthermore, the statement: "It has a configurable standard parallel along which there is no distortion" is a tautology, because the definition of standard parallel is that is a parallel with zero distortion.

When speaking about Werner cordiform projection after the Bonne projection, it will be useful to notice that the Werner projection is a special case of the Bonne projection.

\subsection{Azimuthal}

The title on azimuthal projections in the Map projection article on Wikipedia reads: Azimuthal (projections onto a plane). That suggests that other map projections are not onto a plane! But all map projections are projections into or onto a plane.

Almost any sentence of that chapter contains the term "tangent point". But, the tangent point is in general completely irrelevant for azimuthal projections. First, in perspective projections the location of the plane of projections can be moved to stay parallel, and the image will be larger or smaller in all directions in the same way. Second, in azimuthal projections which are not prespectives, there is no tangent point at all, because the image in the plane of projection can be anywhere in space!

The General Perspective projection can be constructed by using a point of perspective not outside the earth only, but anywhere regarding the sphere: outside, inside or on the sphere (Lapaine, Frančula 1989).

\subsection{Projections by preservation of a metric property}

There is no need that the plane of projection be tangent to the sphere in the stereographic projection. The point of tangency is not important. Classification of map projections into polar, equatorial and oblique is questionable and not recommended. Furthermore, the image of the sphere is limited to a hemisphere (Fig. 5). Who knows the reason? For stereographic projection it should be limited artificially, and it should be explained.

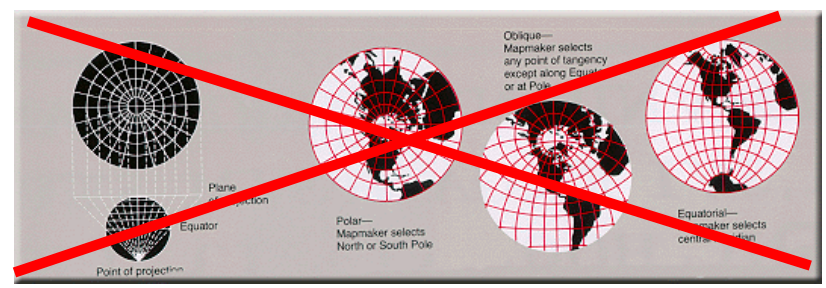

Figure 5. Illustration of stereographic projection. There is no need that the plane of projection be tangent to the sphere. Map maker do not select a point of tangency but the origin of a pseudogeographic coordinate system.

There is Transverse Mercator in the list of conformal projections. It should be added the Gauss-Krüger as another name for the same projection, used in many countries around the world.

In my opinion, as an illustration of equidistant projection can serve Plate carrée and/or azimuthal equidistant instead of two-point equidistant projection of Eurasia.

The definition of Plate carrée projection is rather strange: "an Equirectangular projection centered at the equator". What does it mean "centered at the equator"? For me, any equirectangular projection is centered at the equator. In my opinion, better definition for Plate carrée projection is "equirectangular projection with the equator as a standard line". I will also mention that it is sometimes called the latitude/longitude or lat/lon(g) projection or is said (erroneously) to be "unprojected".

\subsection{Retroazimuthal}

Instead of vertical meridians, I would say parallel meridians in the Craig projection. Verticality is usually connected with horizontality, and in the Craig retroazimuthal projection there is nothing horizontal.

\subsection{Compromise projections}

There is a small list of compromise projections in the Map projection article on Wikipedia. The illustration of the Robinson projection (Fig. 6) is rather strange, and different in comparison with other illustrations in that article. The same map projection is depicted on the left and on the right-hand side of the image. 




Figure 6. Illustration of the Robinson projection, although it remains unclear what the purpose of such an illustration is, because it is the same map projection on the left and on the right-hand side.

Oronce Finé's cordiform map is not produced in the Oronce Finé map projection. The projection should be attributed to Johannes Werner and Johannes Stabius (Pelletier, 1995).

\section{Conclusion}

The present paper analyzed the article Map projection in the English Wikipedia. The author proposed a mathematical approach to map projections because they are based on mathematical formulas. The research has shown that a substantial part of the Wikipedia article Map projection needs to be corrected and improved. This should be done in the near future because people have been increasingly dependent on the Internet and Wikipedia.

\section{Acknowledgements}

The author wish to thank the anonymous reviewers for their valuable comments.

\section{References}

Biernacki, F. (1949, 1965). Teoria odwzorowań powierzchni dla geodetów i kartografów: Warsaw, Główny Urząd Pomiarów Kraju, Prace Geodezyjnego Instytutu Naukowo-Badawczego, no. 4. Polish. Translated into English as Theory of representation of surfaces for surveyors and cartographers: U.S. Dept. of Commerce, 1965

Bugayevskiy L. M. (1998). Matematicheskaya kartografiya, Moscow, Zlatoust, p. 400 (In Russian)

Downs, R., Liben, L. (1991). The Development of Expertise in Geography: A Cognitive Developmental Approach to Geographic Education. Annals of the Association of American Geographers 82: 304-327. doi: 10.1111/j.1467-8306.1991.tb01692.x

Euler, L. (1777). De repraesentatione superficiei sphaericae super plano, Acta Academiae Scientiarum Imperialis Petropolitanae. Translated into German in: Drei Abhandlungen uber Kartenprojection, Ostwald's Klassiker der exakten Wissenschaften, no. 93, 3-37, Leipzig, Wilhelm Engelmann, 1898. Translated into Polish in: Biernacki (1949). Translated into Russian: Ob izobrazhenii poverhnosti shara na ploskosti, Trudy Petersburgskoy akademii nauk za 1777 g., Tom 1, pp. 107-132, in: L. Ejler: Izbrannye kartograficheskhie stat'i, Tri stat'i po matematicheskhoj kartografii, Izdatel'stvo geodezicheskhoj literatury, 1959, Moscow. Translated into English in: Biernacki (1965). Translated into Croatian in: Lapaine (2014)

Kavrayskiy V. V. (1958). Izbrannye trudy. V 2 tomah. Tom 2. Vypusk 1. Matematicheskaya kartografiya. Obshchaya teoriya kartograficheskih proekcij. Moscow, izd. Upravleniya nachal'nika Gidrograficheskoj sluzhby VMF, 680 p. (In Russian)

Lapaine, M. (2014). Kartografske projekcije i njihove deformacije (in Croatian), Peti hrvatski kongres o katastru, Zagreb, 8-9. 5. 2014., Zbornik radova, ISBN 978-953-97081-9-9, 15-32

Lapaine, M. (2017a). Standard Parallel and Secant Parallel in Azimuthal Projections, Cartography and Geoinfor-mation / Kartografija i geoinformacije, Vol. 16, No. 28, 73-88

Lapaine, M. (2017b). Standard Parallels and Secant Parallels in Conic Projections, SJCE, Scientific Journal of Civil Engineering, Skopje, Vol. 6, No. 2, 127-134

Lapaine, M., Frančula, N. (1989). Perspective Projection of the Earth - A General Approach. Presented at the 14th International Cartographic Conference, Budapest, 17-24. 8. 1989. Abstract: Problems in the Automatization of General Perspective Projections. Published in: Abstracts of the 14th World ConferenceICA. Budapest: Hungarian National Committee, ICA, 630-631

Lapaine, M., Frančula, N. (2016). Map projection aspects, International Journal of Cartography, 1-21, doi: 10.1080/23729333.2016.1184554

Lee, L. P. (1944). The nomenclature and classification of map projections. Empire Survey Review. VII (51): 190200. doi:10.1179/sre.1944.7.51.190

Miller, O. M. (1942). Notes on cylindrical world map projections: Geographical Review, Vol. 32, No. 3, 424430

Pelletier, M. (1995). Die herzförmige Weltkarte von Oronce Fine, 1536, Cartographica Helvetica, Sonderheft 9

Serapinas B. B. (2005). Matematicheskaya kartografiya: Uchebnik dlya vuzov. Moscow, izd. Akademiya, 336 p. (In Russian)

Snyder, J. P. (1977). A comparison of pseudocylindrical map projections. Amer. Cartographer, Vol. 4, No. 1, 59-81

Snyder, J. P., Voxland Ph. M. (1989). Album of Map Projections, U.S. Geological Survey Professional Paper 1453

Snyder J. P. (1993). Flattening the Earth: Two Thousand Years of Map Projections. University of Chicago Press

USGS (2000). Map Projections. U.S. Department of the Interior, U.S. Geological Survey, 509 National Center, Reston, VA 20192, USA, URL: http://rnlnx635.er.usgs. gov/mac/isb/pubs/MapProjections/projections.html, Last modified 28 Dec 2000, Accessed 29 Oct 2017 
Vakhrameeva L. A., Bugayevskiy L. M., Kazakova Z. L. (1986). Matematicheskaya kartografiya. Moscow, izd. Nedra, 289 p. (In Russian).

Wayback Machine (2014). Map Projections: From Spherical Earth to Flat Map. https://web.archive.org/ web/20140826044613/http://nationalatlas.gov/articles/m apping/a_projections.html, Accessed 3 Nov 2017

Weisstein EW (2017). "Flat Surface." From MathWorldA Wolfram Web Resource. http://mathworld.wolfram. com/FlatSurface.html, Accessed 3 Nov 2017

Wikipedia (2018a). Reliability of Wikipedia, https://en. wikipedia.org/wiki/Reliability_of_Wikipedia (Accessed 25 Nov 2018)

Wikipedia (2018b). Map projection, https://en.wikipedia. org/wiki/Map_projection (Accessed 25 Nov 2018)

Wikipedia (2018c). Geometry, https://en.wikipedia.org/ wiki/Geometry (Accessed 25 Nov 2018)

Wikipedia (2018d). Sphere, https://en.wikipedia.org/wiki/ Sphere (Accessed 25 Nov 2018)

Wikipedia (2018e). Ellipsoid, https://en.wikipedia.org/ wiki/Ellipsoid (Accessed 25 Nov 2018) 